\title{
Comparando autoavaliação e avaliação de professores sobre as habilidades sociais de crianças com deficiência mental ${ }^{12}$
}

\author{
Lucas Cordeiro Freitas ${ }^{3}$ \\ Zilda Aparecida Pereira Del Prette ${ }^{4}$ \\ Universidade Federal de São Carlos
}

\begin{abstract}
Resumo
O presente estudo investigou a validade convergente do Sistema de Avaliação de Habilidades Sociais (SSRS-BR), em uma amostra de crianças brasileiras com deficiência mental, por meio da correlação entre as subescalas e escalas globais comuns aos formulários de autoavaliação e de avaliação pelos professores. Participaram 84 crianças com deficiência mental de uma escola especial e seus professores, que responderam ao SSRS-BR. Os resultados apontaram que na escala global de habilidades sociais e nas subescalas comuns, não houve correlação significativa entre os dois tipos de avaliadores. Além disso, os estudantes apresentaram uma tendência sistemática de se autoavaliaram mais negativamente em relação à avaliação dos professores. $\mathrm{O}$ estudo apontou para uma possível limitação na utilização do questionário de autoavaliação do SSRS-BR como instrumento único de avaliação no caso de crianças com deficiência mental. A pesquisa sugere ainda questões adicionais de cuidado no uso e comparação de resultados de diferentes informantes.
\end{abstract}

Palavras-chave: habilidades sociais; deficiência mental; autoavaliação; avaliação por professores.

\begin{abstract}
This study investigated the convergent validity of the Social Skills Rating System (SSRS-BR) in a sample of Brazilian children with mental disabilities. Correlations were tested between the commons global scale and subscales from the forms of selfevaluation and evaluation by teachers. Participants 84 were children with mental disabilities from a special school and their teachers, who responded the SSRS-BR. The results showed no significant correlation between the two types of evaluators in the social skills global scale and the common subscales. In addition, the students showed a systematic tendency to evaluate themselves more negatively compared with their teachers. The study pointed a possible limitation in the use of self-evalution questionnaire as single assessment procedure for children with disabilities. The research also suggests additional caution in the use and comparison of results from different evaluators.
\end{abstract}

Keywords: social skills; mental disabilities; self-evaluation; teacher evaluation.

\footnotetext{
${ }^{1}$ Este artigo apresenta parte dos resultados da dissertação de mestrado do primeiro autor, sob orientação da segunda autora. $\mathrm{O}$ estudo teve $\mathrm{o}$ apoio financeiro da FAPESP.

2 Endereço para correspondência: Lucas Cordeiro Freitas, Rua Argentina, 461, apt. 38, Bairro Nova Estância. São Carlos - SP, CEP: 13.566-600. E-mail: 1cordeirofreitas @ yahoo.com.br.

${ }^{3}$ Graduado em Psicologia (Formação de Psicólogo e Licenciatura) pela Universidade Federal de São João del-Rei. Mestre e Doutorando em Educação Especial pela Universidade Federal de São Carlos.

${ }^{4}$ Professora Titular da Universidade Federal de São Carlos. Pesquisadora com bolsa 1A do CNPq. Doutora em Psicologia Experimental pela Universidade de São Paulo com pós-doutorado pela Universidade da Califórnia.
} 
De acordo com Del Prette e Del Prette (2005), as habilidades sociais podem ser definidas como as diferentes classes de comportamentos sociais do repertório de um indivíduo que contribuem para sua competência social, tornando o seu relacionamento com as demais pessoas mais saudável e produtivo. Segundo Kelly (2002), as habilidades sociais têm três funções principais para um indivíduo, quais sejam: de facilitar o estabelecimento de relações com outras pessoas, de alcançar reforçadores que, por si só, não são de natureza social e de impedir que as demais pessoas restrinjam o reforçamento ao qual o indivíduo tem direito.

A aprendizagem das habilidades sociais ocorre inicialmente na infância, basicamente por meio de três processos comportamentais - a modelação (aprendizagem vicariante), o estabelecimento de regras e o manejo das conseqüências (reforçamento e punição) - que são predominantemente mediados por agentes educacionais, tais como os pais e professores (Del Prette \& Del Prette, 2005).

A preocupação com a promoção das habilidades interpessoais na Educação em geral e na Educação Especial em particular, pode ser justificada por seu potencial papel em diminuir os conflitos entre os alunos, amenizar as dificuldades de aprendizagem e ainda preparar a criança para a vida em sociedade, dentro e fora do contexto escolar (Del Prette \& Del Prette, 2006, 2005, 2003, 1998). No caso específico de crianças com deficiência mental, a avaliação e promoção de habilidades sociais pode ser um componente importante para a adaptação escolar, uma vez que essa população apresenta dificuldades interpessoais associadas ao comprometimento no funcionamento intelectual (Luckasson et al., 2002; Freitas \& Del Prette, 2010a).

Tendo em vista as atuais políticas públicas de inclusão de alunos com deficiência mental no ensino regular, a avaliação de suas habilidades sociais se torna ainda mais relevante. Alguns autores defendem que essas habilidades podem ser uma variável facilitadora do processo de integração e inclusão de deficientes mentais na escola (Del Prette \& Del Prette, 1998; Fuchs \& Fuchs, 1994; Heward 2003). Dessa forma, o desenvolvimento de estudos que investiguem o papel das habilidades sociais na inclusão escolar depende criticamente de indicadores comportamentais confiáveis para a avaliação do repertório social dos alunos que participam desse processo.

Nesse contexto, surge a necessidade da elaboração e validação de instrumentos de avaliação do repertório social especificamente voltados para crianças com deficiência mental em idade escolar. O presente trabalho se insere em um conjunto mais amplo de 
estudos que visam à validação e normatização para o Brasil de um instrumento de medida americano para múltiplos informantes (estudantes, pais e professores), o Social Skills Rating System (Gresham \& Elliott, 1990) ou Sistema de Avaliação de Habilidades Sociais (SSRS-BR). Esta pesquisa deu continuidade à verificação das qualidades psicométricas do instrumento, iniciada por Bandeira, Del Prette, Del Prette e Magalhães (2009), por meio do estudo de sua validade convergente em uma amostra de crianças com deficiência mental.

Em Psicometria, a validade convergente pode ser definida como a relação significativa entre duas ou mais medidas de um mesmo construto ou de construtos teoricamente relacionados, utilizando-se diferentes métodos ou instrumentos de avaliação (Pasquali, 2003). No caso do SSRS-BR, diferentes métodos podem ser definidos como diferentes fontes de avaliadores (alunos, pais e professores) (Gresham \& Elliott, 1990). Uma medida de validade convergente, portanto, seria a extensão em que diferentes avaliadores fazem uma avaliação similar de um mesmo indivíduo. No caso do presente estudo, a validade convergente na amostra de crianças com deficiência mental foi avaliada por meio da correlação entre as subescalas e escalas globais comuns aos formulários de autoavaliação dos estudantes e o formulário respondido pelos professores.

\section{Método}

Inicialmente, o projeto foi aprovado pelo Comitê de Ética em Pesquisa da UFSCar (Parecer CEP no 309/2006, Anexo 4) e seguiu suas devidas recomendações. Os pais das crianças participantes do estudo assinaram um Termo de Consentimento Livre e Esclarecido, autorizando a participação de seus filhos.

\section{Participantes}

Participaram do estudo 84 crianças com deficiência mental leve, moderada ou inespecificada, estudantes de uma escola especial do interior do estado de São Paulo, Brasil, com idades entre oito e 14 anos (Média=11,64; d.p.=1,47). Do total de participantes, $56(66,7 \%)$ eram meninos e $28(33,3 \%)$ eram meninas.

Os estudantes foram selecionados para a pesquisa a partir dos resultados obtidos em uma avaliação multidisciplinar padrão, realizada pela própria instituição, que 
atestava o seu diagnóstico de deficiência mental. Essa avaliação foi baseada em anamnese com os pais das crianças, avaliação fonoaudiológica, pedagógica, psicológica e, eventualmente, de acordo com a necessidade, avaliações por neurologista e terapeuta ocupacional. O diagnóstico de deficiência mental, assim como a especificação de seu grau de severidade, foram realizados de acordo com os critérios do DSM-IV (Manual Diagnóstico e Estatístico de Transtornos Mentais, 4a edição, 2002), com auxílio das informações obtidas na avaliação das crianças. Sessenta e nove crianças $(82,1 \%)$ da amostra possuíam deficiência mental leve, 12 (14,3\%) possuíam grau moderado e apenas três $(3,6 \%)$ não tinham grau especificado de deficiência.

Participaram ainda, como informantes, dez professoras dos alunos selecionados, com idade média de 43,33 anos (d.p.=12,66), sendo que nove delas possuíam formação de nível superior e apenas uma possuía formação de segundo grau.

\section{Instrumentos de Avaliação}

\section{Inventário SSRS-BR}

Para a avaliação do repertório social e acadêmico das crianças foi utilizada a versão brasileira do Sistema de Avaliação das Habilidades Sociais (SSRS-BR - Social Skills Rating System) (Bandeira, Del Prette, Del Prette \& Magalhães 2009.). O SSRSBR se encontra validado para o Brasil para crianças com desenvolvimento típico e com deficiência mental e apresenta propriedades psicométricas adequadas de validade de construto, consistência interna e estabilidade temporal (Bandeira et al., 2009; Freitas \& Del Prette, 2010b). O instrumento possui ainda bons indicadores de validade de critério, conforme o estudo de Freitas e Del Prette (2010a).

Este inventário avalia as habilidades sociais, os problemas de comportamento e a competência acadêmica de crianças do ensino fundamental, por meio de três questionários, dirigidos aos pais, aos próprios estudantes e aos professores. Na presente pesquisa, foram utilizados somente os questionários dos estudantes e dos professores, uma vez que avaliam as habilidades sociais mais diretamente relacionadas ao contexto escolar. 
Formulário para Estudantes: Este formulário é composto somente pela Escala de Habilidades Sociais, com 35 itens avaliados em termos de frequência e distribuídos em seis fatores: responsabilidade, empatia, assertividade, autocontrole, evitação de problemas e expressão de sentimento positivo. As alternativas de respostas estão dispostas em uma escala tipo Likert, que varia de 0 a $2(0=$ nunca, $1=$ algumas vezes e $2=$ muito frequente).

Formulário para Professores: O formulário para professores possui três escalas que avaliam as habilidades sociais, os problemas de comportamento e a competência acadêmica dos estudantes, descritas a seguir.

Escala de Habilidades Sociais: Esta escala contém 30 itens avaliados em termos da frequência e da importância das habilidades sociais das crianças: para a frequência, as alternativas de resposta estão dispostas em uma escala que varia de 0 a $2(0=$ nunca, $1=$ algumas vezes e $2=$ muito frequente) e para a importância, em escala de 0 a $2(0=$ não importante, $1=$ importante e $2=$ muito importante). A análise fatorial desta escala para professores indicou a presença de cinco fatores para a escala de habilidades sociais: responsabilidade/cooperação, asserção positiva, autocontrole, autodefesa e cooperação com pares (Bandeira et al., 2009).

Escala de Problemas de Comportamento: Esta escala é composta por 18 itens que avaliam a frequência de problemas de comportamento, cujas alternativas de resposta estão dispostas em uma escala que varia de 0 a $2(0=$ nunca, $1=$ algumas vezes e $2=$ muito frequente). A análise fatorial do questionário para professores indicou a presença de dois fatores para esta escala: problemas externalizantes e problemas internalizantes (Bandeira et. al., 2009).

Escala de Competência Acadêmica: Esta escala possui nove itens que avaliam a competência acadêmica dos estudantes por meio de cinco alternativas de respostas, que visam classificar os alunos com relação à sua turma, da seguinte maneira: $1=10 \%$ piores, $2=20 \%$ piores, $3=40 \%$ médios, $4=20 \%$ bons e $5=10 \%$ ótimos. A análise fatorial do questionário para professores indicou a presença de apenas um fator para a escala de competência acadêmica (Bandeira et al., 2009).

Deve-se destacar que no presente estudo foram utilizadas somente as Escalas de Habilidades Sociais das versões para professores e estudantes do SSRS-BR. 


\section{Procedimento}

\section{Coleta de Dados}

A aplicação do SSRS-BR nas crianças foi realizada, individualmente, em salas disponibilizadas pela coordenação da escola. A forma de aplicação do instrumento seguiu o mesmo padrão da validação do instrumento para crianças sem deficiência (Bandeira et. al., 2009), porém com algumas pequenas modificações. Nas sessões de aplicação, o pesquisador estabeleceu breve rapport com as crianças, fez a leitura das questões do instrumento, solicitou as respostas e as anotou. Diferentemente da aplicação realizada no estudo inicial de validação, o aplicador utilizou folhas com as alternativas de respostas, escritas em números e letras com grandes caracteres, para facilitar a resposta das crianças. $\mathrm{O}$ aplicador lia as questões em tom de voz pausado e solicitava a resposta por parte da criança. Em alguns casos, a criança simplesmente apontava a alternativa escolhida na folha de resposta e em outros, a criança verbalizava a opção escolhida. Se a criança demorasse um tempo considerável para responder alguma questão ou levantasse dúvida, o aplicador repetia a leitura da questão e solicitava novamente a resposta da criança.

As professoras responderam um questionário para cada aluno de sua classe que participou da pesquisa e os mesmos foram devolvidos ao pesquisador em um intervalo médio de 20 dias. Para isso, foram realizadas breves reuniões com cada uma delas, para fornecer as instruções de preenchimento dos questionários e esclarecer as suas dúvidas. Após esse contato, as professoras preenchiam os questionários em horários livres na própria escola ou em casa, conforme sua disponibilidade.

\section{Análise dos dados}

Os dados coletados foram digitados em um banco de dados no programa SPSSPC (Statistical Program for Social Sciences), versão 10.0, para a realização de análises estatísticas. A validade convergente do instrumento foi testada por meio da correlação de Pearson entre os escores do instrumento de avaliação pela criança e de avaliação pelo professor. Foi adotado como nível de significância um valor de $\mathrm{p}<0,05$. 


\section{Resultados}

A Tabela 1 apresenta as médias, desvios-padrão e as correlações entre as subescalas e escalas globais comuns entre os questionários de avaliação pelas crianças e de avaliação pelo professor.

Tabela 1. Médias, desvios-padrão e correlações entre as subescalas e escalas globais comuns entre os questionários de avaliação pelas crianças e pelo professor.

\begin{tabular}{clcccc}
\hline $\begin{array}{c}\text { Escala global e } \\
\text { subescalas de } \\
\text { Habilidades Sociais }\end{array}$ & Avaliadores & Média & $\begin{array}{c}\text { Desvio- } \\
\text { padrão }\end{array}$ & $\begin{array}{c}\text { Correlação } \\
r\end{array}$ & $\begin{array}{c}\text { Significância } \\
p\end{array}$ \\
\hline Escala Global & Estudante & 31,80 & 7,93 & 0,11 & 0,31 \\
& Professor & 37,03 & 9,69 & & \\
\hline Subescala & Estudante & 10,57 & 2,76 & 0,08 & 0,49 \\
Responsabilidade & Professor & 21,14 & 5,83 & & \\
\hline Subescala & Estudante & 7,75 & 2,64 & 0,20 & 0,08 \\
Assertividade & Professor & 10,17 & 3,70 & & \\
\hline Subescala & Estudante & 3,99 & 1,80 & 0,01 & 0,94 \\
Autocontrole & Professor & 10,33 & 3,74 & & \\
\hline
\end{tabular}

Pode-se notar que, quanto à escala global de habilidades sociais, não houve correlação significativa entre a avaliação da criança e a avaliação do seu professor (r= $0,11 ; \mathrm{p}=0,31)$. Esse mesmo padrão foi observado com relação às subescalas de Responsabilidade $(r=0,08 ; p=0,49)$, Assertividade $(r=0,20 ; p=0,08)$ e Autocontrole $(r=$ $0,01 ; \mathrm{p}=0,94)$, em que não houve correlação significativa entre os dois tipos de avaliadores.

Em todas as escalas e subescalas comuns de avaliação, os estudantes apresentaram uma tendência sistemática de se autoavaliaram de maneira mais negativa em relação à avaliação dos professores. Todas as médias nas escalas de autoavaliação foram menores em comparação com as escalas de avaliação pelo professor. 


\section{Discussão}

Estudos anteriores demonstram que o SSRS-BR tem apresentado boas propriedades psicométricas para a avaliação das habilidades sociais em crianças brasileiras (Bandeira et. al., 2009; Freitas \& Del Prette, 2010a; Freitas \& Del Prette, 2010b). No entanto, os resultados deste estudo apontaram que tanto na escala global de habilidades sociais, quanto nas subescalas de Responsabilidade, Assertividade e Autocontrole, não houve correlação significativa entre os dois tipos de avaliadores: estudantes e professores.

Deve-se ressaltar que, apesar de as subescalas comuns de auto-avaliação e avaliação pelo professor medirem as mesmas dimensões do construto (Responsabilidade, Assertividade e Autocontrole), alguns itens não são equivalentes nas duas versões do instrumento, o que pode contribuir para a falta de correspondência entre as duas avaliações.

No estudo de validação da versão original do SSRS-BR (Gresham \& Elliott, 1990), foram encontradas correlações significativas entre as escalas e subescalas comuns de avaliação por estudantes e professores. No entanto, ainda que significativas, as correlações encontradas foram baixas, com média de $r=0,20$. Além disso, deve-se destacar que a amostra de validação do instrumento original era composta por crianças com e sem indicativos de necessidades educacionais especiais, o que pode ter contribuído para uma correlação significativa entre a avaliação de crianças e professores.

No presente estudo, em todas as escalas e subescalas comuns de avaliação, os estudantes apresentaram uma tendência sistemática de se autoavaliaram de maneira pior em relação à avaliação dos professores, sugerindo, de acordo com Gresham, Lane, MacMillah, Bocian e Ward (2000), um viés negativo na autoavaliação de habilidades sociais. Esse tipo de viés se caracteriza por uma autoavaliação da criança substancialmente mais negativa em comparação a avaliações realizadas por outros informantes, como os professores (Gresham, Lane, MacMillah, Bocian \& Ward, 2000).

Del Prette e Del Prette (2006) apontam que a falta de correlação entre a autoavaliação da criança e a avaliação de adultos pode refletir a existência de falhas de ajustamento social da criança ao seu ambiente, assim como déficits na habilidade de automonitoria, considerada indispensável para o aprimoramento da competência social. 
Pode-se supor, ainda, que a habilidade de automonitoria seja mais deficitária no caso de crianças com deficiência mental, levando-se em conta suas desvantagens sociais e cognitivas. O déficit na habilidade de automonitoria, portanto, pode, em parte, explicar a tendência das crianças com deficiência mental se auto-avaliarem de maneira mais negativa que os seus professores.

A ausência de correlação significativa entre a avaliação da criança com deficiência mental e a avaliação do professor sobre o seu repertório de habilidades sociais aponta para uma possível limitação na utilização do questionário de autoavaliação do SSRS-BR como instrumento único de avaliação no caso dessas crianças. Esse resultado evidencia a necessidade da utilização de uma metodologia multimodal de avaliação (Del Prette \& Del Prette, 2006), principalmente em crianças que apresentam comprometimentos nas habilidades acadêmicas. A utilização simultânea dos instrumentos de auto-avaliação e de avaliação por professores ou pais é uma vantagem do SSRS-BR que não deve ser negligenciada na avaliação de crianças com necessidades educacionais especiais.

Gresham e Elliott (2008) assinalam que a baixa correlação entre avaliadores pode indicar que a criança se comporta de forma diferente em contextos distintos, que os avaliadores percebem as habilidades sociais e os problemas de comportamento de forma diferenciada, ou uma combinação desses dois fatores. Nessa mesma direção, a presente pesquisa indica questões adicionais de cuidado no uso e comparação de resultados de diferentes informantes.

Sugere-se que futuras pesquisas sejam realizadas utilizando também a avaliação dos pais sobre o repertório de habilidades sociais de suas crianças com deficiência mental. A exemplo de estudos realizados com crianças com problemas de comportamento, a visão dos pais sobre as habilidades sociais de seus filhos nem sempre converge com a avaliação feita pelo professor (Dias, 2010; Bolsoni-Silva, Marturano, Pereira \& Manfrinato, 2006), indicando a necessidade de se investigar, com maior refinamento, os critérios de julgamento utilizados por diferentes informantes. 


\section{Referências}

American Psychiatric Association (2002). Diagnostic and statistical manual of mental disorders. ( $4^{\mathrm{a}}$ ed.). Washington: APA.

Bandeira, M., Del Prette, Z.A.P., Del Prette, A., \& Magalhães, T. (2009). Validação das escalas de habilidades sociais, comportamentos problemáticos e competência acadêmica (SSRS-BR) no ensino fundamental. Psicologia: Teoria e Pesquisa, 25 (2), 271-282.

Bolsoni-Silva, A.T., Marturano, E.M., Pereira, V.A. \& Manfrinato J.W.S. (2006). Habilidades sociais e problemas de comportamento de pré-escolares: comparando avaliações de mães e de professoras. Psicologia: Reflexão e Crítica, 19 (3), 460469.

Del Prette, Z.A.P. \& Del Prette, A. (1998). Desenvolvimento interpessoal e educação escolar: O enfoque das habilidades sociais. Temas em Psicologia, 6 (3), 205-215.

Del Prette, Z.A.P. \& Del Prette, A. (2003). Habilidades sociais e dificuldades de aprendizagem: Teoria e pesquisa sob um enfoque multimodal. Em A. Del Prette \& Z.A.P. Del Prette (Org.), Habilidades sociais, desenvolvimento e aprendizagem (pp. 167- 206). Campinas: Alínea.

Del Prette, Z.A.P. \& Del Prette, A. (2005). Psicologia das habilidades sociais na infância: Teoria e prática. Petrópolis: Vozes.

Del Prette, Z.A.P. \& Del Prette, A. (2006). Avaliação multimodal de habilidades sociais em crianças: Procedimentos, instrumentos e indicadores. Em: Bandeira, M., Del Prette, Z.A.P. \& Del Prette, A. (Orgs.). Estudos sobre habilidades sociais $e$ relacionamento interpessoal (pp. 47-68). São Paulo: Casa do Psicólogo.

Dias, T.P. (2010). Observação versus relato de mães e professoras sobre competência social e comportamentos problemáticos em pré-escolares. Dissertação de Mestrado, Programa de Pós-Graduação em Psicologia, Universidade Federal de São Carlos. São Carlos, SP.

Freitas, L.C. \& Del Prette, Z.A.P. (2010a). Validade de critério do Sistema de Avaliação de Habilidades Sociais (SSRS-BR). Psicologia: Reflexão e Crítica. Artigo aceito para publicação. 
Freitas, L.C. \& Del Prette, Z.A.P. (2010b). Validade de construto do Sistema de Avaliação de Habilidades Sociais para crianças brasileiras com deficiência intelectual. Interamerican Journal of Psychology. Artigo aceito para publicação.

Fuchs, D. \& Fuchs, L.S. (1994). Inclusive schools movement and the radicalisation of special education reform. Exceptional Children, 60, 294-309.

Gresham, F.M. \& Elliott, S.N. (1990). Social Skills Rating System: Manual. Circle Pines, MN: American Guidance Service.

Gresham, F.M. \& Elliott, S.N. (2008). Social skills improvement system: Rating scales. Bloomington: Pearson Assessments.

Gresham, F.M., Lane, K.L., MacMillan, D.L., Bocian, K. \& Ward, S. (2000). Effects of positive and negative illusory biases: Comparisons across social and academic selfconcept domains. Journal of School Psychology, 38, 151-175.

Heward, W.L. (2003). Exceptional children: An introduction to special education. (7 ed.). New Jersey: Merril Prentice Hall.

Kelly, J.A. (2002). Entrenamiento de las habilidades sociales: Guia práctica para intervenciones. $7^{\mathrm{a}}$ ed. Bilbao: Desclée de Brouwer.

Luckasson, R., Borthwick-Duffy, S., Buntinx, W.H.H., Coulter, D.L., Craig, E.M., Reeve, A. \& Snell, M.E. (2002). Mental Retardation: Definition, classification, and systems of support. Washington: American Association on Mental Retardation.

Pasquali, L. (2003). Psicometria: Teoria dos testes na Psicologia e na Educação. Petrópolis: Editora Vozes. 